\title{
Caracterización del saber pedagógico: Estudio en profesorado novel
}

\section{Characterization of Pedagogical Knowledge: A Study of Novice Teachers}

Alejandro Almonacid-Fierro' Universidad Autónoma de Chile

Facultad de Educación

Talca, Chile

aalmonacidf@uautonoma.cl

Eugenio Merellano-Navarro² Universidad Autónoma de Chile

Facultad de Educación

Talca, Chile

emerellanon@uautonoma.cl

Alberto Moreno-Doña ${ }^{3}$

Pontificia Universidad Católica de Valparaíso

Valparaíso, Chile

amorenodona@gmail.com

Recibido 9 de diciembre de 2013 • Corregido 14 de mayo de 2014 • Aceptado 22 de agosto de 2014

\footnotetext{
${ }^{1}$ Doctorando en Ciencias de la Educación de la Universidad de Sevilla, España. Magister en Educación de la Universidad Diego Portales. Magister en Motricidad Humana mención Educación de la Universidad Autónoma de Chile. Profesor de Educación Física de la Pontificia Universidad Católica de Chile. Docente asociado de la Universidad Autónoma de Chile, sede Talca. Experiencia como investigador en el área de formación del profesorado.

${ }^{2}$ Magister en Motricidad Humana mención en Educación de la Universidad Autónoma de Chile. Profesor de Educación Física de la Universidad Católica Silva Henríquez de Chile. Docente asistente de la Universidad Autónoma de Chile, sede Talca. Experiencia como investigador en el área de la didáctica de la educación física.

${ }^{3}$ Doctor en Actividad Física para la Educación en la Sociedad del Conocimiento, Universidad de Granada, España. Magister Interdisciplinario en Estudios Latinoamericanos, mención Filosofía, Universidad de la Serena. Profesor de Educación Física, Universidad de Valencia. Docente de la Pontificia Universidad Católica de Valparaíso, Chile. Experiencia como investigador en las áreas democratización y formación de profesores y pedagogía crítica e institución escolar.
} 
doi: http://dx.doi.org/10.15359/ree.18-3.10

URL: http://www.una.ac.cr/educare

CORREO: educare@una.cr

Resumen. El saber pedagógico como un despliegue específico de las narrativas y configuraciones paradigmáticas e ideológicas sobre el carácter y sentido de la acción educativa es, en definitiva, lo que cada docente explicita como ambiente de aprendizaje. De ahí que su caracterización y categorización se convierta en un eje central de conocimiento científico y pedagógico. El propósito del presente artículo científico fue indagar, describir e interpretar cuáles son las relaciones entre la práctica desplegada en el aula y los saberes pedagógicos desde la relación docente-discente en profesores noveles ${ }^{4}$ egresados de la Facultad de Educación de la Universidad Autónoma de Chile. Se utilizó la metodología cualitativa, sustentada en la perspectiva epistemológica del paradigma interpretativo optando por la comprensión subjetiva de dicho proceso. Quienes participaron enfatizaron la importancia de la experiencia práctica como algo fundamental en su formación docente. Adicionalmente, indicaron como positivo el énfasis que otorga la Universidad Autónoma de Chile en la búsqueda de un personal docente reflexivo, crítico y autónomo a través de una práctica pedagógica con énfasis en la co-construcción del conocimiento.

Palabras claves. Formación docente, profesores noveles, saber pedagógico.

Abstract. Pedagogical knowledge, understood as a specific display of paradigmatic and ideological narratives and configurations about the character and sense of the educational action, is ultimately what each teacher defines as the learning environment. Therefore, its characterization and categorization become a central concept of scientific and pedagogical knowledge. The main objective of this article was to investigate, describe and interpret the relationship between the practice shown in the classroom and the pedagogical knowledge from the teacher-learner relationship in novice ${ }^{5}$ teachers graduated from the School of Education of Universidad Autónoma de Chile. A qualitative research approach was used, based on the epistemological perspective of an interpretative paradigm, utilizing the subjective comprehension of the process. Participants emphasized the importance of practical experience as fundamental in their teacher training. In addition, they see positively the emphasis given by Universidad Autónoma de Chile on forming reflective, critical and autonomous teachers through a teaching practice focused on the co-construction of knowledge.

Keywords. Teacher education, novice teachers, pedagogical knowledge.

El presente artículo es parte de una investigación mayor, que busca comprender e interpretar las relaciones entre la práctica desplegada y el saber pedagógico de docentes que han egresado de la Facultad de Educación de la Universidad Autónoma, sede Talca.

Enesta primera instancia, exponemos la caracterización del saber pedagógico de profesores noveles y su despliegue en el ámbito escolar, reconociendo los procesos de formación inicial de profesorado como una instancia que favorece la mediación entre el conocimiento disciplinar y el conocimiento pedagógico.

La problemática del saber pedagógico, construido desde las prácticas escolares, considera el aprendizaje de un corpus de conocimientos articulado y situado desde la relación

\footnotetext{
${ }^{4}$ Se entiende por profesores noveles, aquellos docentes que cuentan con menos de 5 años de ejercicio profesional en el sistema escolar.

${ }^{5}$ Novice teachers are those who have less than five years of professional experience in the school system.
} 
dialógica entre teoría y práctica; comprende aspectos pedagógicos, disciplinares, profesionales y prácticos de la profesión docente, cuestión que se traduce, finalmente, en la emergencia desde el punto de vista sistémico- de un saber que orienta el desempeño profesional, pero que en dicho proceso se dinamiza, crea y re-crea con contradicciones y perplejidades (Ibáñez, 2008).

No obstante, y dado lo situado del fenómeno educativo (Toro y Niebles, 2013), dicho saber se diferencia y distingue en las relaciones específicas originadas en contextos definidos y se desenvuelve de acuerdo con las características que actores y contexto hacen confluir.

Para la comprensión de nuestra problemática, nos referiremos a las diversas instancias formativas y como estas favorecen la emergencia de los saberes pedagógicos que experimenta el profesorado novel de las diversas carreras pertenecientes a la Facultad de Educación de la Universidad Autónoma de Chile. Creemos relevante profundizar este tema porque, si bien las instituciones educativas posibilitan la realización de cursos destinados a promover un proceso educativo centrado en la co-construcción de los aprendizajes, la realidad nos muestra cómo el proceso didáctico y pedagógico sigue sustentado en el paradigma tradicional, generando una concepción positivista de la enseñanza y un modelo pedagógico marcadamente conductista que, centrándose en un lógica cartesiana, separa linealmente los diferentes elementos que conforman el proceso de conocer (Almonacid, 2011; Castro, Correa y Lira, 2006; Magendzo, 2008; Pinto, 2008).

El saber pedagógico es propio de la pedagogía, toda vez que el profesorado es, o al menos debiera ser, portador de un saber construido, entre otros espacios-tiempos, durante su proceso de formación inicial, y que, a su vez, comparte durante su ejercicio profesional en las aulas escolares. El saber, propiamente tal, es una noción compleja, pues se conforma de aspectos curriculares, prácticos y reflexivos que se relacionan e interactúan desde la totalidad. En consecuencia, no existe separación o fragmentación, dado que el fenómeno educativo se despliega, operacionalmente, en una relación de carácter sistémico y multidireccional (Alliaud y Vezub, 2012; Barròn, 2006; Francis y Marín, 2010; Ibáñez, 2008; Mancovsky y Monetti, 2012; Tardif, 2004).

En este sentido, el saber docente se comprende en íntima relación con su trabajo en la escuela y en el aula. Es decir, aunque el profesorado utilice diferentes saberes, esa utilización se da en función de su trabajo y de las situaciones, condicionamientos y recursos ligados a esa responsabilidad. En suma, el saber está al servicio del trabajo y desde ahí se actualiza y reconfigura. "Las relaciones de los docentes con los saberes no son nunca unas relaciones estrictamente cognitivas; son relaciones mediadas por el trabajo que les proporciona unos principios para afrontar y solucionar situaciones cotidianas" (Tardif, 2004, p. 14).

De esta manera, el saber pedagógico construido y, posteriormente, compartido por el profesorado en su labor docente puede ser entendido como un saber plural formado por una amalgama, más o menos coherente, de conocimientos provenientes de la formación profesional, que incorporan áreas disciplinarias, curriculares, pedagógicas y prácticas. En consecuencia, nuestro problema de investigación considera tres tipos de saberes que configuran al personal docente en su quehacer profesional. 
doi: http://dx.doi.org/10.15359/ree.18-3.10

URL: http://www.una.ac.cr/educare

CORREO: educare@una.cr

Cuando nos referimos al saber curricular, hablamos de aquellos saberes que la universidad intenciona en sus estudiantes durante el proceso de formación inicial y que les permite desarrollar una serie de competencias profesionales y académicas útiles para la inserción en el mundo laboral. Por su parte, el saber experiencial se basa en el trabajo cotidiano y en el conocimiento que el profesor posee del medio escolar; surge de la experiencia, que se encarga de validarlos. Se incorpora a la experiencia individual y colectiva en forma de hábitos y habilidades, de saber hacer y de saber ser. Finalmente, desde la dimensión teórica y práctica, emerge un saber reflexivo que se recupera o construye desde la conciencia, en los continuos procesos de análisis crítico de la realidad escolar que el personal docente desarrolla de manera sistemática a partir de la realidad del sistema escolar y las prácticas pedagógicas asociadas (Aristizábal, 2006; Barròn, 2006; Cárdenas, Soto-Bustamante, Dobbs-Díaz, Bobadilla-Goldschmidt, 2012; Diaz, 2006; Francis y Marín, 2010; Tardif, 2004; Terigi, 2012).

Una vez explicitados cuales son y cómo se construyen los saberes pedagógicos, desarrollamos, brevemente, la perspectiva que nos permite identificar las características de lo que se define como 'buen personal docente'. Este se caracteriza por ser profesional capaz de conocer y aplicar un currículo que, como país, ha sido considerado positivo tras la selección cultural que como construcción conlleva. A partir de aquí, se infiere que la formación docente debe estar acorde con lo que nacionalmente se está entendiendo como con educación de calidad. Se reconoce, entonces, que la universidad debe brindarle, tras la selección del saber erudito, la posibilidad de desarrollar las competencias del saber conocer y del saber hacer.

Conforme el profesorado novel se inserta en el mundo laboral, va enriqueciendo y complejizando los conocimientos y competencias construidas en el proceso universitario (Tardif, 2004); es decir, el saber construido está íntimamente relacionado tanto con la identidad del sujeto como con el contexto en que se desenvuelve. Lo anterior, en el entendido de que la perspectiva desde la que nos situamos corresponde al paradigma de la complejidad que, en palabras de Morin (2005), "provendrá del conjunto de nuevos conceptos, de nuevas visiones, de nuevos descubrimientos y de nuevas reflexiones que van a conectarse y reunirse ... el principio de la complejidad, se fundará sobre la predominancia de la conjunción compleja" (p. 110), puesto que los elementos que configuran el ethos docente, vale decir, la historicidad, el currículo, la didáctica, la concepción epistemológica, la relación pedagógica y un largo etc., se ligan y religan en un proceso recursivo, dialógico y hologramático, para permitir la emergencia del saber pedagógico.

Desde lo planteado hasta, aquí la investigación realizada tiene como objetivo la comprensión de la caracterización de los saberes pedagógicos construidos en el profesorado novel de la Facultad de Educación de la Universidad Autónoma de Chile, en sus procesos de formación profesional. Se entiende este comienzo en la vida profesional del personal docente como una etapa de múltiples tensiones generada por experimentar las diversas complejidades que representa dicha tarea. Esta etapa de adquisición de saberes en la experiencia escolar está llena de inseguridades que, en ocasiones, son angustiantes; producto del choque 
doi: http://dx.doi.org/10.15359/ree.18-3.10

URL: http://www.una.ac.cr/educare

CORREO: educare@una.cr

de realidades entre lo vivenciado en las aulas universitarias y el contexto profesional real: comprende que, en el despliegue en el aula, no basta con enseñar, sino que se debe aprender a enseñar a estudiantes (Álvarez, 2008; Barba, 2011; Barròn, 2006; Buenfil, 2011; Díaz, 2006; Erazo, 2011; Ribot, 2006).

\section{Modelo educativo y pedagógico de la Universidad Autónoma de Chile}

El modelo que implementa la Universidad Autónoma de Chile se orienta hacia el desarrollo de capacidades constituidas por conocimientos, habilidades, destrezas, actitudes y valores que, al igual que las competencias, se conciben como actuaciones integrales ante actividades y problemas del contexto con idoneidad y compromiso ético (Tobón, 2010).

Cuenta con 4 ejes que se articulan, de manera recíproca e interdependientemente, en los modelos pedagógicos y curriculares, y como parte integral del sistema de aseguramiento de la calidad de los procesos académicos. Estos ejes son:

1. Centralidad del estudiantado: no se postula traspasar al estudiantado la responsabilidad de guiar su proceso formativo, sino que, se responsabilice, progresivamente, y asuma el protagonismo de su proceso formativo, pero con el acompañamiento constante del personal docente.

2. Aprendizaje transversal: consiste en entender al currículo como proyecto formativo integrado, en el que los aprendizajes transversales están llamados a constituir puntos de encuentro entre lo disciplinar y lo pedagógico formativo humano, de manera que se logre la integralidad del aprendizaje.

3. Aprendizaje continuo: promueve la formación permanente, considerando que la velocidad de cambio mundial, en las sociedades actuales, demanda una actualización de los conocimientos para poder interactuar, social y críticamente en un mundo cada vez más competitivo.

4. Responsabilidad social universitaria: habilidad y efectividad de la Universidad para responder a las necesidades de transformación de la sociedad donde está inmersa, mediante el ejercicio de las funciones sustantivas: docencia, investigación y extensión. Estas funciones deben estar animadas por la búsqueda de la promoción de la justicia, la libertad y equidad, mediante la construcción de respuestas exitosas para atender los retos que implica promover el desarrollo.

Desde este modelo, desarrollado a modo de síntesis, la Facultad de Educación de la Universidad Autónoma de Chile es una comunidad académica comprometida con el aprendizaje en diversos contextos y su principal propósito es ser un agente de cambio educativo. Se espera que el futuro o futura profesional, al egresar de la Facultad, tenga un conocimiento profundo, contextual y actualizado de su disciplina. Por lo tanto, estará preparado o preparada para 
doi: http://dx.doi.org/10.15359/ree.18-3.10

URL: http://www.una.ac.cr/educare

CORREO: educare@una.cr

enfrentar un entorno cambiante, desarrollando habilidades y actitudes personales tales como la capacidad de trabajo colaborativo, autoestima, flexibilidad y valoración de la diversidad. Se comunica de manera efectiva en diversas situaciones asociadas a su quehacer, evidenciando juicio profesional que le permite reflexionar sobre su práctica y su inserción en el sistema educativo.

\section{Metodología}

En el presente estudio se pretende no solo conocer la realidad sino que, principalmente, la intención es comprenderla. Y es así porque la comprensión lleva implícita la generación de conocimientos sobre la práctica educativa para transformarla y mejorarla. Es decir, "que el mismo proceso de investigación se convierta en proceso de aprendizaje de los modos, contenidos, resistencias y posibilidades de la innovación de la práctica en el aula conforme a los valores que se consideran educativos" (Pérez, 1999, p. 117). La idea central es indagar en los significados que los sujetos le otorgan a los fenómenos educativos (Ghiso, 2006; Murcia y Jaramillo, 2008; Pérez, 1999; Rodríguez, Gil y García, 1999; Ruiz, 2003; Sandín, 2003).

Se adopta una metodología cualitativa, toda vez que la investigación referida a saberes pedagógicos del profesorado egresado de la Universidad Autónoma pretende acercarse a la comprensión subjetiva que tienen los sujetos en relación con un hecho, suceso, situación, temática, símbolos y objetos determinados, vale decir, como ellos significan, representan $y$, de alguna manera, dan sentido a los saberes aprendidos en su formación inicial y luego transmitidos en su ejercicio profesional (Davila, 1999; Flores, 2009; Gibbs, 2012; Rodríguez et al. 1999; Vasilachis, 2006).

Se utilizaron entrevistas en profundidad y grupos focales. Los sujetos participantes (ver tabla 1) fueron seleccionaros a través de los siguientes criterios (Flores, 2009).

- Accesibilidad: Profesorado egresado de la Universidad Autónoma que trabaja en la Región del Maule.

- Representación: Las características que debían poseer los sujetos de estudio fueron las siguientes:

- Profesional titulado de la Universidad y perteneciente a alguna de las carreras de la Facultad de Educación.

- Que trabajen en dependencias: municipal, particular subvencionado o particular pagado.

- Años de egreso 2008 a 2012.

- Sexo: Hombre o mujer

- Interés en participar en el estudio. 
Tabla 1

Detalle de grupos focales y entrevistas realizadas

\begin{tabular}{lcc}
\hline \multicolumn{1}{c}{ Carrera } & Grupo focal & Entrevista en profundidad \\
\hline Pedagogía en Arte & 1 & 1 \\
Pedagogía en Educación Básica & - & 2 \\
Pedagogía en Matemáticas & 1 & 1 \\
Pedagogía en Educación Física & 1 & 1 \\
Pedagogía en Historia y Geografía & 1 & 1 \\
Pedagogía en Inglés & - & 2 \\
Pedagogía en Castellano & 1 & 1 \\
Pedagogía en Educación Parvularia & 1 & 1 \\
\hline
\end{tabular}

\section{Proceso de recolección y análisis de la información}

Una vez definidos los sujetos, se citaron a una reunión en las dependencias de la Universidad Autónoma de Chile. Se realizaron 10 entrevistas y 6 grupos focales en horarios distintos y con seis participantes en cada uno de dichos grupos.

Posteriormente se llevaron a cabo las etapas de transcripción, categorización de la información, sistematización, análisis y reducción de datos. "El proceso de análisis de datos se realizó con un procedimiento inductivo, utilizando como técnica el análisis de contenido, cuyo primer paso consiste en la codificación de los datos, para luego construir la matriz de sistematización" (Castillo y Almonacid, 2012 p. 673).

Para iniciar el proceso de categorización, en primer término, recurrimos al marco teórico, toda vez que desde estos referentes es posible determinar las categorías previas que nos permiten situar la problemática investigativa, para seguidamente iniciar el proceso de codificación, como lo presenta Coffey y Atkinson (2003, p. 32): "Ios códigos vinculan diferentes segmentos o ejemplos presentes en los datos. Traemos estos fragmentos de los datos y los reunimos para crear categorías que definimos con base en alguna propiedad". Posteriormente se agrupan las categorías previas a través de la codificación selectiva (Strauss y Corbin, 2002), cuestión que permite la emergencia de las categorías primarias, dando paso, desde un primer momento descriptivo, a una instancia de carácter interpretativo.

Lo anterior orienta la comprensión que el profesorado novel otorga a la caracterización de los saberes pedagógicos, desde la red de significaciones propias de estos sujetos, en un proceso de conocer sus experiencias y vivencias a través del diálogo que se despliega desde el discurso de los propios protagonistas. Discurso que, como investigadores, trabajamos en términos de 
doi: http://dx.doi.org/10.15359/ree.18-3.10

URL: http://www.una.ac.cr/educare

CORREO: educare@una.cr

datos cualitativos a través de un ejercicio de sistematización, optando por la propuesta de la teoría fundamentada (Strauss y Corbin, 2002).

Los datos de la investigación se presentan en el siguiente orden (ver tabla 2), en una primera parte, categorías previas y, posteriormente, categorías primarias con su respectivo descriptor y los relatos más significativos de cada una de ellas.

Tabla 2

Matriz sistematización

\begin{tabular}{|c|c|c|c|}
\hline Megacategoría & Categorías previas & Descriptor & Categoría primaria \\
\hline \multirow{4}{*}{$\begin{array}{l}\text { Saberes desplegados } \\
\text { por estudiantado } \\
\text { egresado de la Facultad } \\
\text { de Educación UA }\end{array}$} & Tipos de saberes & $\begin{array}{l}\text { Categoría que alude } \\
\text { a los tres dominios de } \\
\text { este saber: reflexivo, } \\
\text { laboral y conceptual }\end{array}$ & $\begin{array}{l}\text { - Saber reflexivo } \\
\text { - Saber curricular o conceptual } \\
\text { - Saber laboral o experiencial }\end{array}$ \\
\hline & $\begin{array}{l}\text { Aprendizajes en la } \\
\text { formación inicial }\end{array}$ & $\begin{array}{l}\text { Saberes pedagógicos, } \\
\text { emergencia en el } \\
\text { transcurso de la línea } \\
\text { de formación práctica }\end{array}$ & $\begin{array}{l}\text { - Reflexión en la práctica } \\
\text { - Aspectos o ámbitos disciplinares } \\
\text { - Métodos de enseñanza y aprendizaje } \\
\text { - Ejercicio profesional } \\
\text { - Desarrollo humano } \\
\text { - Formación pedagógica } \\
\text { - Diversidad en el área educativa }\end{array}$ \\
\hline & & & $\begin{array}{l}\text { - Enfocadas en el currículo } \\
\text {-Enmarcadas en el área de formación } \\
\text { práctica }\end{array}$ \\
\hline & $\begin{array}{l}\text { Sugerencias para } \\
\text { la formación del } \\
\text { profesorado }\end{array}$ & $\begin{array}{l}\text { Propuestas para } \\
\text { la mejora de la } \\
\text { formación docente }\end{array}$ & $\begin{array}{l}\text { - Relacionada con el proceso de } \\
\text { formación } \\
\text { - Relacionada con el profesorado } \\
\text { - Relacionada con la comunicación } \\
\text { - Relacionada con la evaluación }\end{array}$ \\
\hline
\end{tabular}

Nota: Matriz que ejemplifica la totalidad de categorías investigadas. En este artículo solo se trabajara la primera categoría previa:Tipos de saberes. 


\section{Resultados y discusión sobre la categoría previa tipos de saberes Categoría primaria 1: Saber reflexivo}

Sí... nosotros tenemos momentos que llamamos horas de reajuste curricular y que podemos utilizarlas para corregir pruebas, realizar guías, pruebas... En mi caso yo lo utilizo para hacer mi reflexión pedagógica ... Cuando me doy cuenta, a través de las pruebas, de que hay un contenido que no fue adecuadamente abordado por los niños, me preocupo de retomarlos para que, finalmente, se pueda adquirir ese aprendizaje de manera significativa ... (Marta: tiempos pedagógicos para reflexionar).

La mejora del conocimiento y de la acción docente se debe sustentar en el proceso de la investigación personal, es decir, como sujeto pensante, el personal docente es capaz de descubrir y ser consciente de su accionar, ideas que se han puesto de manifiesto desde la formación del profesorado en los procesos de formación inicial, pues se hace necesario que en dichos procesos se reconozca a los futuros profesores y profesoras como sujetos de conocimientos, los cuales debiesen generar una estructura curricular practico-activa y reflexiva.

En lo que hoy se ha denominado comunidad escolar, se han otorgado instancias de mejora al profesorado, generadas a partir del dialogo con diferentes actores que intervienen en los procesos educativos. Sin embargo, la connotación e importancia que se da al reajuste curricular ${ }^{6}$ en el contexto escuela permite al profesorado mejorar su quehacer pedagógico. Por lo tanto, es pertinente considerar las horas de reajuste curricular, instancias de conversación y tiempos de análisis sobre el accionar que permite generar en el contexto escuela un proceso reflexivo que promueve la reflexión. El objetivo es que la reflexión repiense las experiencias vividas para aportar así a la construcción de conocimiento profesional, es decir, ser consciente de su propia acción pedagógica. La reflexión crítica sobre la práctica se torna una exigencia de la relación teórica/práctica sin la cual la teoría puede convertirse en palabrería y la practica en activismo. Es preciso insistir nuevamente en que el pensar crítico $^{7}$ se genera desde una óptica basada en la teoría de la complejidad, a través de esta postura emerge la relación analítica con el que hacer práctico.

Por lo tanto, profesionales de la educación, seres pensantes, críticos e investigativos de su accionar, debiesen generar conocimiento e instancias de aprendizaje; pero no conocimientos absolutos e irrevocables, sino conocimientos sujetos a un constante cambio, generados en un contexto auténtico en el que se promueve la relación entre la teoría y la práctica. Por eso, las

\footnotetext{
${ }^{6}$ Se entiende por reajuste curricular al proceso de diagnóstico detallado del currículo nacional mediante consultas a diversos actores educativos. Actualmente, el nuevo currículo ya se está implementando.

7 Proceso cognitivo que consiste en la reorganización del conocimiento a través del análisis y la evaluación de lo que se que pretende interpretar. Pensamiento de orden superior junto al pensamiento creativo.
} 
doi: http://dx.doi.org/10.15359/ree.18-3.10

URL: http://www.una.ac.cr/educare

CORREO: educare@una.cr

mejoras y nuevas estrategias que emergieran en los procesos de construcción de conocimiento son generadas por los procesos reflexivos, los cuales van desde la acción práctica a una reflexión y vuelven nuevamente a la práctica.

Debido a que los procesos de formación de profesorado han estado fuertemente influenciados por una lógica aplicacionista y una visión fragmentada, se puede observar una enseñanza basada en la oralidad, un bajo trabajo práctico y una escasa vinculación con la realidad educativa y las demandas del contexto; razón por la cual se hace necesario que en dicho proceso se reconozca al futuro profesorado como sujeto de conocimiento con quien se debiese generar, desde la formación inicial del profesorado, una estructura curricular practica, activa y reflexiva.

Bueno... si no hay reflexión no hay cambio o no existe la capacidad de reinventarse como profesor... nosotros como educadores debemos ir modificando nuestras estrategias constantemente, todo porque nuestros alumnos también cambian. (Pedro: transformación como resultado de la reflexión).

Entonces, los procesos reflexivos constantes permiten al personal docente tener la plasticidad de cambio constante, de poder moldearse a estrategias y nuevos conocimientos generados por la conciencia de su actuar, facilitando, además, aprendizajes situados al contexto a través de una didáctica emergente de la relacionalidad de sujetos en función de los aprendizajes. Desarrollando las habilidades que le permiten configurar al personal docente como un ser autónomo, generador de conocimiento, favorecedor de aprendizajes significativos en sus estudiantes y agente que mejora la calidad del proceso educativo.

... podemos entender que la reflexión constante permite mejoras en el proceso enseñanzaaprendizaje, tal importancia debe generar... mejorías al docente en formación y más bien al docente inserto en el mundo profesional-laboral. Es súper importante porque te permite darte cuenta en qué posición estas y cómo puedes mejorar y cambiar actividades... (Juan: reflexión como proceso de concientización).

Sin embargo, la escuela posee una poderosa influencia socializadora, creando concepciones pedagógicas que tienden a reproducirse fácilmente en el futuro profesional docente, debido a que se encuentra una ideología pedagógica dominante, la que es estimulada e incluso exigida por el funcionamiento de las instituciones. Con ello se potencia el desarrollo y proliferación de prácticas uniformes, rutinarias y estereotipadas, exigidas de alguna manera para la reproducción. Esto conlleva una fragmentación de los contenidos del currículo y una escasa vinculación del proceso de formación con la realidad o con los requerimientos del contexto (Almonacid, 2011; Maturana, 1999; Morin, 2001, Morin, Roger y Motta, 2003). 


\section{Categoría primaria 2: Saber curricular o conceptual}

Depende mucho de la línea del colegio...en el caso de nosotros...hay un departamento que... tiene una línea de trabajo... Se consideran los lineamientos de los planes y programas, pero es el colegio es el que adapta... en este caso hay trabajos particulares de cada uno... depende de la línea del colegio, más de lo que uno puede proponer. Eh... el toque que uno le va a dar a la clase entorno a los contenidos que a uno le den pero siento yo que eso uno lo fija de una u otra manera el colegio... (María: uso de referentes curriculares).

El despliegue en sus aproximaciones en el aula, sumado a la interacción con sus pares y estudiantes, y el proceso de formación académica vivido en la Universidad otorgan, al profesorado, los saberes pedagógicos necesarios para desempeñarse exitosamente en el contexto escolar. Estos saberes son regidos por agentes externos que condicionan y regularizan los contenidos que serán entregados a sus estudiantes, como por ejemplo: la administración del colegio, regularizados por el PEI (Proyecto Educativo Institucional), UTP (Unidad Técnico Pedagógica) o principalmente por el Ministerio de Educación y sus planes de estudio. A través de esta influencia, gran parte del despliegue que realiza en el aula esta sobrecargado por lo que estos agentes quieren y no con lo que realmente necesitan aprender el estudiantado. Por lo tanto, el personal docente no deviene en un productor de saber, sino un trasmisor de saber. Tardif (2004, p. 31) menciona: "La relación que los profesores mantienen con los saberes es la de "trasmisores", "portadores" u "objetos" de saber, pero no productores". Desde este punto de vista, los docentes adquieren sus concomimientos para, posteriormente, entregarlos a sus estudiantes bajo el alero de modelos lineales que lo limitan a ser un trasmisor de conocimiento más que productor de su propio saber; todo ello muy ajeno a la creación y legitimación de su acción. Para poder evidenciar lo expuesto, presentamos el siguiente relato:

... estoy trabajando en una escuela municipal. Principalmente nos guiamos por el libro que entrega el gobierno, pero a mi parecer este libro realmente no está acorde con la realidad que poseen nuestros alumnos, al trabajar en un colegio municipal el Ministerio te da prácticamente todo el material... te entrega los planes y programas, contenidos mínimos obligatorios todo..., ahora ingles sufrió un reajuste en cuanto a sus bases curriculares y de hecho yo trabajo con todo el material, también trabajo con el de la escuela propia que han ido dejando otros colegas que han estado antes que yo ... Se trabaja en conjunto, siempre junto a la jefa de UTP...(Fabiola: utilización de los planes y programas del ministerio de educación).

Si bien es cierto, los docentes reciben de parte del Ministerio de Educación los contenidos por enseñar, en ciertos casos estos planes y programas de estudio parecen no estar contextualizados a la realidad de todo el estudiantado, porque en sus bases de creación toman un solo punto de vista, generalizando y alejándose a la realidad diversa y compleja de los centros educativos a lo largo del país. 
doi: http://dx.doi.org/10.15359/ree.18-3.10

URL: http://www.una.ac.cr/educare

CORREO: educare@una.cr

\section{Categoría primaria 3: Saber laboral o experiencial}

Uno puedeobservar distintas realidades en los colegios, tanto particulares, subvencionados o municipales, pero al momento de hacer una clase... es distinto, uno puede saber mucho, pero si no dominas un curso, nunca vas a poder hacer una buena clase (Raúl: dominio de grupo).

En el ejercicio docente, los profesores van adquiriendo saberes específicos necesarios y característicos en el medio en el que se desenvuelven. El dominio del conocimiento de del entorno se adquiere a través de las relaciones personales y la experiencia en el medio laboral docente, por lo tanto, las vivencias forman al experto o experta y, como consecuencia de estas vivencias, emergen los saberes de ser y hacer, los cuales la experiencia se encarga de validar.

En la práctica docente existen miles de circunstancias diferentes que pueden influir en la relación del profesorado con sus estudiantes y con sus pares. Realidades tan generales como posibilidades existen, siendo en estas contingencias (de fácil o difícil resolución) en donde el profesorado tiene la obligación de saber cómo reaccionar y tratar aquellas situaciones. En este aspecto de la práctica docente es donde la perspectiva teórico-práctica adquiere la connotación que se merece, ya que el dominio de diversas situaciones mediante el criterio subjetivo no es algo enseñable, más bien es adquirido personalmente con la vivencia, donde posiblemente un escenario similar se vuelva a repetir.

En Tardif (2004, p. 80) se define el saber experiencial como: "Es un saber práctico; su utilización depende de su adecuación a las funciones, problemas y situaciones peculiares del trabajo. La cognición del profesor está, por tanto, condicionada por su actividad".

Por lo tanto, el saber cómo reaccionar o qué hacer en ciertas circunstancias requiere de un saber práctico, habilidad de adecuarse a la adversidad con maestría, un dominio del conocimiento abstracto materializado en una acción concreta que requiere de decisiones específicas.

La estrategia es que el departamento de lenguaje trabaja de forma paralela... esto quiere decir, que por ejemplo... yo tengo un quinto y la otra persona también tiene un quinto y tenemos que ir a la par es decir compartimos las planificaciones, obviamente uno va, como dijimos antes, se va adaptando al contexto y a la realidad del curso, pero nosotros trabajamos en conjunto, entonces es esa la estrategia que utilizamos... como departamento trabajar en paralelo (Andrés: comparte las planificaciones).

Claramente el apoyo entre el profesorado es de beneficio mutuo, este se ve reflejado en sus estudiantes y en la equidad en cuanto a los aprendizajes. Esta práctica es un ejemplo de las interrelaciones que existen entre docentes, ya sea de una misma área o de áreas diferentes. 
El saber experiencial "es un saber interactivo, movilizado y modelado en el ámbito de las interacciones entre docentes y otros agentes educativos" (Tardif, 2004, p. 80). Esto alude a la normatividad, desde el punto de vista afectivo; recurre a procedimientos diversos de interpretación de situaciones rápidas, inestables y complejas que, de cierto modo, no son posibles de manejar individualmente y requieren el apoyo y la interacción de otros individuos para su solución.

Claramente la experiencia toma un papel protagónico en el buen desempeño docente, pues cada experiencia de vida suma conocimientos muy valiosos que le permitirán adquirir las herramientas necesarias para enfrentar iguales o similares situaciones y desarrollarlas, teniendo como telón de fondo los referentes de la teoría pedagógica y la educación.

El trabajo docente está propenso a sufrir muchos cambios, por tanto, el maestro o maestra tiene que adaptarse y reinventarse permanentemente, debe integrar lo nuevo y adherirse al acervo de un maestro comprometido, donde el conocimiento será una necesidad vital que las vivencias traen consigo, requiriendo solo la reflexión para arrebatarlas.

Yo me considero responsable, a pesar de que quizás ... dejo el día sábado para descansar, y el día domingo tener que planificar y hacer material, entonces, dejo todo para el ... domingo, pero a pesar de todo llevo mis cosas el día como corresponde ... me considero responsable porque llevo todas mis cosas, me considero alegre, porque soy como una niña más que se tira al suelo, que salta, que patalea, que grita, que hace todo y soy cariñosa con ellos y a veces soy enojona. (Andrea: Estrategias curriculares)

Cada docente desprende en su accionar su esencia, lo que hace que algo sea lo que es. La esencia es el carácter, el que está constituido por nuestras actitudes que reflejan lo que somos y lo que pretendemos ser. El carácter está condicionado por las vivencias, donde cada situación vivida conlleva a un aprendizaje especifico propio de lo experiencial. Por lo tanto, el saber experiencial es un saber existencial, pues no solo está ligado a la experiencia de trabajo, sino también a la historia vital del profesorado, a lo que fue y a lo que es, lo que significa que está incorporado a la propia vivencia del personal docente, a su identidad, a su hacer y a sus maneras de ser.

La experiencia laboral de un docente no engloba el cúmulo de saberes adquiridos por un profesor, ya que, a lo largo de la vida, han existido vivencias que han dejado un aprendizaje especifico, incluso vivencias tan significativas que condicionan el carácter del sujeto.

Inconscientemente, a la hora de ejercer la labor docente, los profesores recurren a replicar modelos de su formación pasada, considerando las cosas que creen a su parecer estar bien y, si consideran que algo estuvo mal, lo mejoran; pero siempre existe la necesidad de recordar y reflexionar sobre sus propias experiencias de vida, extrayendo lo bueno y dejando lo malo, nunca desde cero. 
doi: http://dx.doi.org/10.15359/ree.18-3.10

URL: http://www.una.ac.cr/educare

CORREO: educare@una.cr

Las identidades de los docentes, al inicio de la labor profesional, están determinadas por lo que se ha denominado el "aprendizaje sedimentado", construido a partir de las experiencias escolares como estudiante y de lo que socialmente se les ha dicho que significa ser profesor o profesora (Rivas, 2014, p. 489). Pero este aprendizaje sedimentado se construye y reconstruye a partir de las experiencias que ese mismo profesorado comienza a tener en el espacio de la práctica educativa, una vez egresado de la formación universitaria. Estas experiencias (saber experiencial) van configurando "identidades emergentes" que permiten y facilitan re-pensar el quehacer profesional (Rivas, 2014).

Dentro de las características que presenta el saber experiencial se encuentra la generación de nuevos aprendizajes emergentes desde las experiencias vividas, producto de un constante proceso evolutivo del aprendizaje, ciclo que nunca se detiene y modifica sus esquemas mentales con el fin de lograr algo distinto.

Primero en el tema pedagógico, sin duda... el manejar conceptos que tienen que ver con el ámbito de la educación, como te decía anteriormente son fundamentales, yo creo es la base para insertarse en dentro de los ámbitos educacionales el manejar áreas del currículo, la gestión, considero que todo docente lo deberían tener. Y en ámbito de la didáctica, sin duda hay... cátedras que son una base fundamental, llámese educación física de base, eeh... recreación, que no están tan, tan ligadas al deporte en si, pero si te permite ver en la actividad física o la recreación desde una perspectiva más transversal, en los aprendizajes de los niños" (Ignacio. Currículo y gestión)

Claramente existen cátedras en la formación profesional universitaria que, estando ya insertas en el medio laboral del profesorado, se percatan de la importancia de dominar ciertos contenidos o competencias necesarias para un buen desempeño laboral; o también, en el caso contrario, reconocen aquellas cátedras que no son de una importancia tan práctica en el ámbito laboral profesional. Por lo tanto, el personal docente jerarquiza con base en la experiencia laboral, lo de mayor o menor importancia para sí.

\section{Conclusiones}

El estudio desarrolla la relevancia del saber experiencial en los procesos de formación profesional. Por medio de la experiencia, el profesorado novel genera un conocimiento interactivo, movilizado y modelado por las interacciones entre docentes y estudiantes. Es un saber diverso, donde el conocimiento adquirido facilita el saber hacer en función de realidades variables. Además, es un saber flexible, pues se adquiere a través de experiencias nuevas que se remodelan según las situaciones de trabajo. Asimismo es un saber existencial, ya que está ligado a la historia de vida, a lo que fue y a lo que es quien enseña. Y por último, es un saber temporal, evolutivo y dinámico que se transforma y se construye a lo largo de una historia de vida profesional, dependiendo de una socialización y un aprendizaje de la profesión. Para construir este saber experiencial, los sujetos participantes en esta investigación enfatizan la 
doi: http://dx.doi.org/10.15359/ree.18-3.10

URL: http://www.una.ac.cr/educare

CORREO: educare@una.cr

importancia de los procesos reflexivos como eje articulador para la modificación del quehacer docente a partir del saber experiencial generado. Es desde aquí que los profesores y profesoras se constituyen en profesionales autónomos capaces de contextualizar, según las realidades laborales, sus intencionalidades educativas.

El proceso de desarrollo de los saberes pedagógicos es inacabado y permanente; trasciende instancias formativas entregadas por la universidad. Es producto de una constante reflexión, que conlleva a un ser docente crítico y comprometido con el contexto, de esta forma, los tres saberes analizados se configuran y articulan en un mejoramiento en la calidad de los aprendizajes del aula.

Se realza el rol que posee la universidad en la búsqueda de personal docente reflexivo, se indaga que sea capaz de lograr una autonomía que lo haga darse cuenta, por sí mismo, de qué actividades realizar para el mejoramiento del aprendizaje de sus estudiantes. Por ello, el reflexionar críticamente acerca del actuar favorece el descubrimiento de nuevas formas para aprender y enseñar.

No obstante, la estructura rígida que poseen los planes y programas, y la influencia que se recibe de parte de la institución donde trabaja, hace que el profesorado novel no incluya en sus planificaciones los intereses de sus estudiantes y se vea obligado a enseñar contenidos no adecuados al contexto en el que está inserto. La rigidez comentada viene determinada por una estructura de la institución escolar burocratizada y más ligada al deber ser institucional que a las posibilidades reales de construcción del conocimiento por parte del alumnado. La administración de los colegios, la unidad técnico pedagógica, las jefaturas de departamento, etc., siempre saben qué es lo que los otros deben hacer y así se lo hacen saber al profesorado, lo que provoca que el despliegue de docentes en las aulas esté determinado por estas exigencias y no por lo que verdaderamente necesita el alumnado.

\section{Referencias}

Alliaud, A. y Vezub, L. (setiembre-diciembre, 2012). El oficio de enseñar. Sobre el quehacer, el saber y el sentir de docentes argentinos. Revista Diálogo Educativo, 12(37), 927-952. doi: http://dx.doi.org/10.7213/dialogo.educ.7211

Almonacid, A. (2011). El sistema escuela: Un acercamiento desde el paradigma de la complejidad. Revista Foro Educacional, 19, 13-29.

Álvarez, N. (2008). La situación de los profesores noveles. Madrid: OEI y Fundación SM. Recuperado de http://www.oei.es/pdfs/la_situacin_de_los_profesores_noveles.pdf

Aristizábal, M. (2006). La categoría "saber pedagógico": Una estrategia metodológica para estudiar la relación pedagogía, currículo y didáctica. Revista ltinerantes, 4, 43-48. 
doi: http://dx.doi.org/10.15359/ree.18-3.10

URL: http://www.una.ac.cr/educare

CORREO: educare@una.cr

Barba, J. J. (2011). El desarrollo profesional de un maestro novel en la escuela rural desde una perspectiva crítica (tesis doctoral). Universidad de Valladolid, España. Recuperado de http://uvadoc.uva.es/bitstream/10324/822/1/TESIS96-110331.pdf

Barròn, C. (2006). Los saberes del docente: Una perspectiva desde las humanidades y las ciencias sociales. Revista Perspectiva Educacional, 48, 11-26. Recuperado de http://www.redalyc. org/pdf/3333/333328829001.pdf

Buenfil, R. (2011). [Reseña de libro Otra educación. Aprendizajes sociales y producción de saberes, por M. M Ruiz]. Revista Perfiles Educativos, 33(131), 194-200. Recuperado de http:// www.redalyc.org/articulo.oa?id=13218531012

Cárdenas, A. V., Soto-Bustamante, A. M., Dobbs-Díaz, E. y Bobadilla-Goldschmidt, M. (setiembrediciembre, 2012). El saber pedagógico: Componentes para una reconceptualización. Educación y Educadores, 15(3), 479-496. Recuperado de http://educacionyeducadores. unisabana.edu.co/index.php/eye/article/view/1962

Castillo, F.y Almonacid, A. (julio-setiembre, 2012). Las actividades en la naturaleza en la formación inicial docente: Un acercamiento desde los sentidos. Educação e Pesquisa, 38(3), 668-680. Recuperado de http://www.scielo.br/pdf/ep/v38n3/aop614.pdf

Castro, F., Correa, M. E. y Lira, H. (2006). Currículum y evaluación educacional. Aportes teóricos y prácticos para el quehacer docente en el aula. Hualpén: Universidad del Bío-Bío.

Coffey A y Atkinson P. (2003). Encontrar el sentido a los datos cualitativos. Estrategias complementarias de investigación. Medellín: Universidad de Antioquia.

Davila, A. (1999). Las perspectivas metodológicas cualitativa y cuantitativa en las ciencias sociales: Debate teórico e implicaciones praxeológicas. En J. M. Delgado y J. Gutiérrez (Coord.), Métodos y técnicas cualitativas de investigación en ciencias sociales (pp. 69-95). Madrid. Síntesis.

Díaz, V. (2006). Formación docente, práctica pedagógica y saber pedagógico. Revista Laurus, 12 (Extraordinario), 88-103. Recuperado de http://www.redalyc.org/articulo.oa?id=76109906

Erazo, M. S. (2011). Prácticas reflexivas, racionalidad y estructura en contextos de interacción profesional. Revista Perfiles Educativos, 33(133), 114-133. Recuperado de http://www. revistas.unam.mx/index.php/perfiles/article/view/27908

Flores, R. (2009). Observando observadores: Una introducción a las técnicas cualitativas de investigación social. Santiago: Ediciones Universidad Católica de Chile.

Francis, S. y Marín, P. (mayo-agosto, 2010). Hacia la construcción del saber pedagógico en las comunidades académicas: Un estudio desde la opinión de docentes universitarios. Revista Electrónica Actualidades Investigativas en Educación, 10(2), 1-29. Recuperado de http:// www.redalyc.org/pdf/447/44717910002.pdf 
doi: http://dx.doi.org/10.15359/ree.18-3.10

URL: http://www.una.ac.cr/educare

CORREO: educare@una.cr

Ghiso, A. (2006). Rescatar, descubrir, recrear. Metodologías participativas en investigación social comunitaria. En M. Canales (Coord.), Metodología de Investigación social. Introducción a los oficios (pp. 349-377). Santiago. LOM.

Gibbs, G. (2012). El análisis de datos cualitativos en investigación cualitativa. Madrid: Ediciones Morata.

Ibáñez, N. (2008). Saber pedagógico y práctica docente: Un estudio en aulas de educación parvularia y básica. (Informe final). Santiago: Fondo de Investigación y Desarrollo de la Educación. Ministerio de Educación, Chile.

Magendzo, A. (2008). Dilemas del currículum y la pedagogía. Santiago: Lom Ediciones.

Mancovsky, V. Monetti, E. (julio, 2012). Approche de "la critique pédagogique": Une expérience de réflexion et d'analyse sur notre savoir pédagogique [Acerca de "la crítica pedagógica": Una exerienia de reflexión y análisis sobre nuestro saber pedagógico]. Ponencia presentada en la Biennale Internationale de l' education, de la formation et des pratiques professionnelles. Paris. Recuperado de http://halshs.archives-ouvertes.fr/docs/00/77/59/83/PDF/vmancovsky-e-monetti-com-n-201-238-atelier-13_1355746129427.pdf

Maturana, H. (1999). Emociones y lenguaje en educación y política. Santiago: Lom Ediciones.

Morin, E. (2001). Los siete saberes necesarios para la educación del futuro. Barcelona: Paidos.

Morin, E. (2005). Introducción al pensamiento complejo. Barcelona: Gedisa.

Morin, E., Roger, E. y Motta, R. D. (2003). Educar en la era planetaria. Barcelona: Gedisa.

Murcia, N. y Jaramillo, L. G. (2008). Investigación cualitativa. La complementariedad etnográfica, una guía para abordar estudios sociales. Armenia: Editorial Kinesis.

Pérez, A. I. (1999). Comprender la enseñanza en la escuela. Modelos metodológicos de investigación educativa. En J. Gimeno y A. I. Pérez (Coords.), Comprender y transformar la enseñanza (115-136). Madrid: Editorial Morata.

Pinto, R. (2008). El currículum crítico: Una pedagogía transformativa para la educación Latinoamericana. Santiago: Ediciones Universidad Católica de Chile.

Ribot, S. (2006). [Reseña de libro Construcción del saber pedagógico, por V. Díaz]. Laurus, 12(21), 197-198. Recuperado de http://www.redalyc.org/articulo.oa?id=76102114

Rivas, J. I. (2014). Nuevas identidades en la formación del profesorado: La voz del alumnado. International Journal of Development and Educational Psychology,7(1),487-494. Recuperado de http://riuma.uma.es/xmlui/handle/10630/7449

Rodríguez, G. Gil, J. y García, E. (1999). Metodología de la investigación cualitativa. Málaga: Ediciones Aljibe. 
doi: http://dx.doi.org/10.15359/ree.18-3.10

URL: http://www.una.ac.cr/educare

CORREO: educare@una.cr

Ruiz, J. I. (2003). Metodología de la investigación cualitativa (4º ed.). Bilbao: Universidad de Deusto.

Sandín, M. (2003). Investigación cualitativa en educación. Fundamentos y tradiciones. Madrid: McGraw-Hill.

Strauss, A. y Corbin, J. (2002). Bases de la investigación cualitativa: Técnicas y procedimientos para desarrollar la teoría fundamentada. Medellín: Universidad de Antioquia.

Tardif, M. (2004). Los saberes del docente y su desarrollo profesional. Madrid: Narcea.

Terigi, F. (2012). Los saberes docentes, formación, elaboración en la experiencia e investigación. Buenos Aires: Santillana.

Tobón, S. (2010). Formación integral y competencias. Pensamiento complejo, currículo, didáctica y evaluación. Bogotá: ECOE Ediciones.

Toro, S. y Niebles, A. (2013). Corporeidad y aprendizaje en el contexto de la enseñanza general básica: Comprensión y descripción de los procesos de construcción de conocimiento desde la acción relacional de los actores. Estudios Pedagógicos, 39(1), 269-284. doi: http:// dx.doi.org/10.4067/S0718-07052013000100016

Vasilachis, I. (2006). Estrategias de investigación cualitativa. Barcelona: Gedisa.

\section{Cómo citar este artículo en APA:}

Almonacid-Fierro, A., Merellano-Navarro, E. y Moreno-Doña, A. (setiembre-diciembre, 2014). Caracterización del saber pedagógico: Estudio en profesores noveles. Revista Electrónica Educare, 18(3), 173-190. doi: http://dx.doi.org/10.15359/ree.18-3.10

Nota: Para citar este artículo en otros sistemas puede consultar el hipervínculo "Como citar el artículo" en la barra derecha de nuestro sitio web: http://www.revistas.una.ac.cr/index.php/EDUCARE/index 